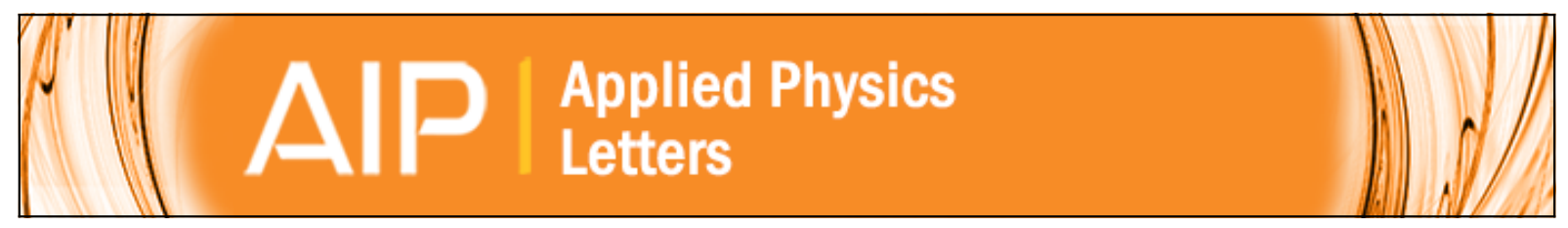

\title{
Luminescence from Si nanocrystals in silica deposited by helicon activated reactive evaporation
}

S. Cheylan, R. G. Elliman, K. Gaff, and A. Durandet

Citation: Applied Physics Letters 78, 1670 (2001); doi: 10.1063/1.1354668

View online: http://dx.doi.org/10.1063/1.1354668

View Table of Contents: http://scitation.aip.org/content/aip/journal/apl/78/12?ver=pdfcov

Published by the AIP Publishing

\section{Articles you may be interested in}

Photoluminescence properties of $\mathrm{SiO} x$ thin films prepared by reactive electron beam evaporation from SiO and silica nanoparticles

J. Appl. Phys. 105, 073517 (2009); 10.1063/1.3104772

Phosphorus ion implantation in silicon nanocrystals embedded in SiO 2

J. Appl. Phys. 105, 054307 (2009); 10.1063/1.3088871

Influence of nitrogen on the growth and luminescence of silicon nanocrystals embedded in silica

J. Appl. Phys. 105, 013108 (2009); 10.1063/1.3054561

Effect of nitride passivation on the visible photoluminescence from Si-nanocrystals

Appl. Phys. Lett. 85, 3408 (2004); 10.1063/1.1787599

The effect of annealing environment on the luminescence of silicon nanocrystals in silica

J. Appl. Phys. 96, 4018 (2004); 10.1063/1.1789265

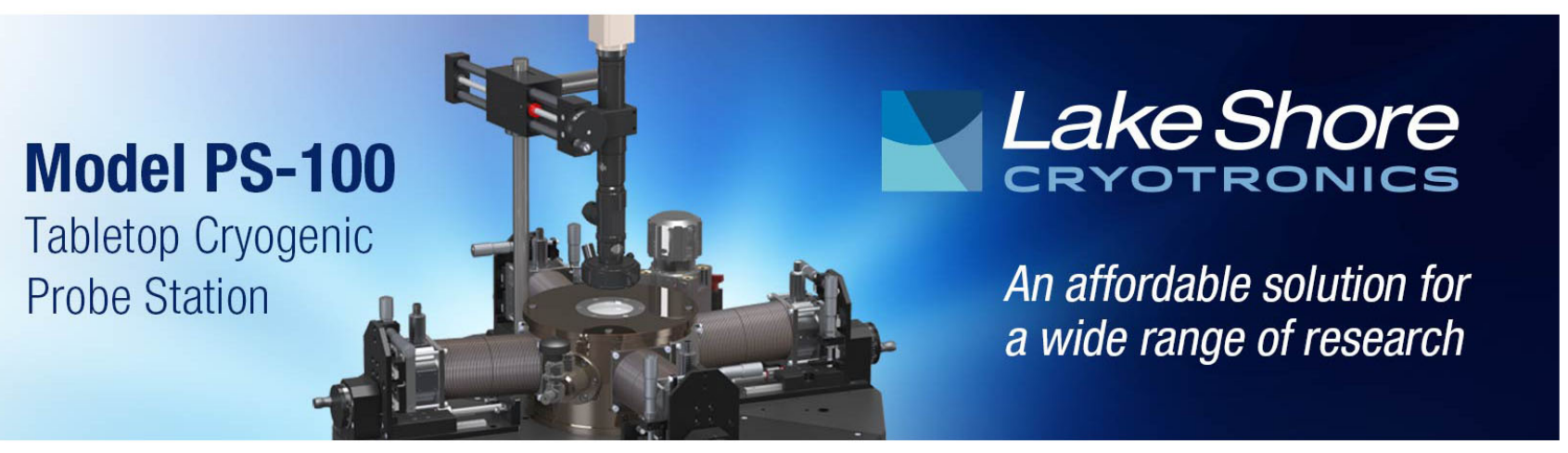




\title{
Luminescence from Si nanocrystals in silica deposited by helicon activated reactive evaporation
}

\author{
S. Cheylan ${ }^{\mathrm{a})}$ and R. G. Elliman \\ Electronic Materials Engineering Department, Research School of Physical Sciences and Engineering, \\ Australian National University, Canberra, ACT 0200, Australia \\ K. Gaff \\ Optical Sciences Center and Plasma Physics Laboratory, Research School of Physical Sciences \\ and Engineering, Australian National University, Canberra, ACT 0200, Australia
}

A. Durandet

Plasma Physics Laboratory, Research School of Physical Sciences and Engineering, Australian National University, Canberra ACT 0200, Australia

(Received 7 July 2000; accepted for publication 16 January 2001)

\begin{abstract}
An alternative method is investigated for the preparation of Si-rich $\mathrm{SiO}_{2}$ films used for the fabrication of light-emitting Si nanocrystal structures. The technique, helicon-activated reactive evaporation (HARE), combines $e$-beam evaporation of silicon with plasma activation of a reactive argon-oxygen atmosphere, and has the advantage of being able to produce thick, H-free films suitable for planar photonic device applications. The nanocrystal-rich films were formed by annealing as-deposited films at $1100^{\circ} \mathrm{C}$ for $1 \mathrm{~h}$. Room temperature photoluminescence was then measured and compared with that from ion-implanted samples annealed under similar conditions. The HARE-deposited films exhibited strong visible luminescence for a range of excess Si concentrations, demonstrating their potential for the manufacture of such materials. The films also exhibited a concentration dependence comparable to that of ion-implanted samples: the luminescence intensity initially increased with excess Si concentration up to a maximum before decreasing with increasing concentration thereafter. The cause of the decrease at higher concentrations is briefly discussed. (C) 2001 American Institute of Physics.
\end{abstract}

[DOI: $10.1063 / 1.1354668]$

Since Canham ${ }^{1}$ first observed strong visible luminescence from porous silicon in 1990, its use for optoelectronic devices has been extensively investigated. Unfortunately, its fragile mechanical properties and unstable luminescence make porous silicon unsuitable for many practical applications. However, nanometre-sized Si crystallites also exhibit strong room temperature luminescence and when embedded in a dielectric layer, such as $\mathrm{SiO}_{2}$, provide a mechanically robust and optically stable material suitable for device applications. There is, therefore, considerable interest in this material system and its optical properties. ${ }^{2-6}$

$\mathrm{Si}$ nanocrystals can be prepared in a variety of ways, including: plasma deposition from silane, ${ }^{7,8}$ silicon-ion implantation into $\mathrm{SiO}_{2},{ }^{2,9}$ crystallization of amorphous silicon, ${ }^{10}$ cosputtering of $\mathrm{Si}$ and $\mathrm{SiO}_{2},{ }^{11}$ reactive ion etching. ${ }^{12}$ In this work, an alternative method, heliconactivated reactive evaporation (HARE $)^{13}$ is employed. This technique combines $e$-beam evaporation of silicon with plasma activation of a reactive argon-oxygen atmosphere by a high density helicon plasma source. The silicon vapor evaporated from a crucible is partially ionized as it passes through the helicon plasma source before condensing on the substrate. The plasma also enhances the reactivity of the atmosphere by ionizing and dissociating oxygen molecules and produces ion bombardment of the growing film to hinder

${ }^{a}$ Electronic mail: smc109@rsphysse.anu.edu.au columnar growth. In this way, high quality silica films can be grown at very low temperatures (typically below $200^{\circ} \mathrm{C}$ ). Importantly, the technique is also capable of producing hydrogen-free films because unlike plasma-enhanced chemical vapor deposition, which typically relies on the decomposition of $\mathrm{SiH}_{4}$ and $\mathrm{N}_{2} \mathrm{O}$, it employs hydrogen free materials. This is important for photonic device applications where $\mathrm{H}$-related absorption can be a limitation and for photoluminescence studies, where the presence of $\mathrm{H}$ is known to strongly influence the luminescence intensity. ${ }^{14}$

This letter compares Si-nanocrystal based photoluminescence (PL) from samples produced by HARE and by ion implantation. Since ion implantation represents one of the most widely used and promising techniques for nanocrystal fabrication, ${ }^{8,14,18}$ a comparison of samples made by these two techniques is a convenient way of assessing the quality of material made by the HARE technique.

Oxygen deficient silica films were grown at approximately $90 \mathrm{~nm} / \mathrm{min}$ in the HARE reactor described in detail elsewhere. ${ }^{13}$ In the present work, silicon was evaporated by a $10 \mathrm{kV}, 0.3 \mathrm{~A}$ electron beam into an argon-oxygen plasma generated by applying $400 \mathrm{~W}$ rf power to the antenna at $13.56 \mathrm{MHz}$. The argon flow rate was kept constant at $3 \mathrm{sccm}$ (corresponding to a pressure of $0.3 \mathrm{mTorr}$ in the process chamber), while the oxygen flow was varied to obtain films with a range of stoichiometries. During deposition the pressure in the process chamber was 0.5 mTorr and the sample temperature was typically less than $200{ }^{\circ} \mathrm{C}^{13}$ The thickness 

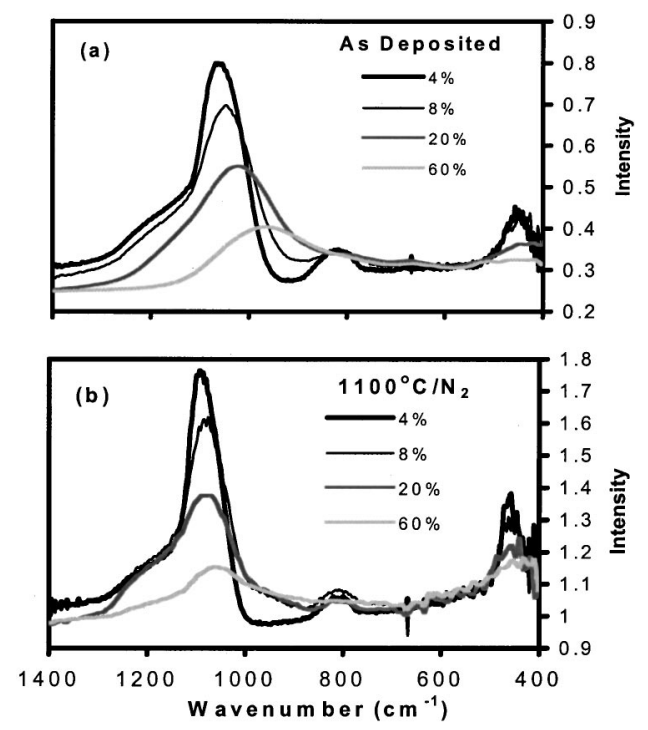

FIG. 1. FTIR spectra of HARE samples with excess Si of 4\%, $8 \%$, 20\%, and $60 \%$. (a) as-deposited samples and (b) after annealing at $1100{ }^{\circ} \mathrm{C}$ for $1 \mathrm{~h}$.

of the films was subsequently checked by ellipsometry and a prism coupler, and was found to be around $500 \mathrm{~nm}$. For comparison, $1 \mu \mathrm{m}$ thick thermal oxide layers, grown on (100)Si wafers, were implanted at room temperature with $400 \mathrm{keV} \mathrm{Si}$ ions to doses in the range from $1 \times 10^{17}$ to 6 $\times 10^{17} \mathrm{~cm}^{-2}$, corresponding to about $5 \%$ to $30 \%$ excess $\mathrm{Si}$, respectively. Both sets of samples were then annealed at $1100{ }^{\circ} \mathrm{C}$ for $1 \mathrm{~h}$ in a $\mathrm{N}_{2}$ ambient to nucleate and grow $\mathrm{Si}$ crystallites.

Samples were measured before and after annealing with Fourier-transform infrared (FTIR) spectroscopy and PL. FTIR measurements were conducted at room temperature using a Nicolet IMPACT 400 model spectrometer operating over the wave number range $400-4000 \mathrm{~cm}^{-1}$. PL spectra were measured at room temperature using the $488 \mathrm{~nm}$ line of an Ar ion laser as the excitation source. The PL emission was collected using a lens to focus the light into a quartz light pipe. This was passed through a single grating monochromator (digikrom model DK480) and detected with a GaAs photomultiplier tube (Hamamatsu R943-02). Standard lock-in detection techniques were used to maximize the signal-to-noise ratio and all spectra were corrected for the system response.

Figure 1 shows FTIR spectra for as-deposited Fig. 1(a) and annealed Fig. 1(b) HARE samples. Three peaks are evident in the spectra, which span the wave number range from 400 to $1600 \mathrm{~cm}^{-1}$. The main absorption peak, located at $1075 \mathrm{~cm}^{-1}$, is associated with the $\mathrm{Si}-\mathrm{O}-\mathrm{Si}$ stretching mode, whilse those at 820 and $475 \mathrm{~cm}^{-1}$ correspond to bending and rocking modes, respectively. For both as-deposited and annealed samples, the absorption decreases with increasing $\mathrm{Si}$ fraction due to the lower fraction of $\mathrm{Si}-\mathrm{O}-\mathrm{Si}$ bonds. The frequency of the main peak also shifts from $1075 \mathrm{~cm}^{-1}$ (stoichiometric $\left.\mathrm{SiO}_{2}\right)$ to $970 \mathrm{~cm}^{-1}(60$ at $\%$ excess $\mathrm{Si})$ as the excess $\mathrm{Si}$ concentration increases, consistent with the increasing probability for $\mathrm{Si}-\mathrm{Si}$ bonds and associated changes in the $\mathrm{Si}-\mathrm{O}-\mathrm{Si}$ stretching frequency. ${ }^{15}$ After annealing, the relative magnitude of the main absorption peaks remains unchanged, reflecting the fact that the stoichiometry is unchanged by annealing (1.e., there is no further oxidation of
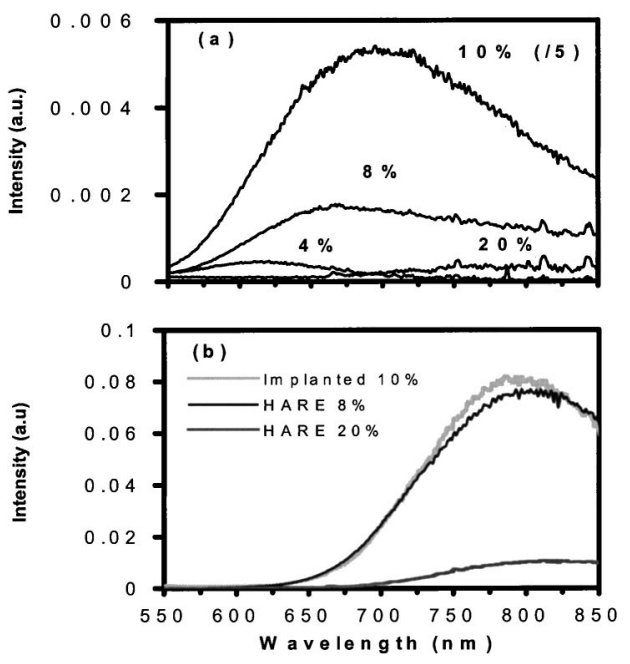

FIG. 2. (a) PL spectra from as-deposited HARE samples having excess $\mathrm{Si}$ concentrations of 4,8 , and 20 at. $\%$ and from an implanted sample with an excess Si concentration of 10 at. \%. Note that the spectrum from the implanted sample is divided by a factor of 5. (b) PL spectra of samples after annealing at $1100{ }^{\circ} \mathrm{C}$ for $1 \mathrm{~h}$. Spectra shown for HARE samples with 8 and 20 at. \% excess Si and for an implanted sample with 10 and 20 at. \% excess $\mathrm{Si}$.

the excess $\mathrm{Si}$ ), but the peaks now show a much smaller shift with increasing Si content. This is consistent with formation of Si precipitates since their formation returns the surrounding material to stoichiometric $\mathrm{SiO}_{2}$. Spectra from implanted silica samples (not shown) do not change significantly with increasing implant dose because the implanted volume represents a small fraction of the $\mathrm{SiO}_{2}$ film thickness.

Figure 2(a) shows PL spectra for as-deposited HARE samples having nominal excess $\mathrm{Si}$ concentrations of 4, 8, and 20 at. $\%$, and for an as-implanted sample with a peak excess Si content of 10 at. $\%$. The spectra from the 8 at. \% HARE sample and the 10 at. \% implanted sample both show a broad emission peak centered around 670-720 nm. This peak has previously been attributed to defect emission in the $\mathrm{SiO}_{2}$ matrix. ${ }^{16}$ Importantly, the maximum defect emission from the HARE samples is much weaker than that from implanted samples, and for excess Si concentrations less than 8 at. \% it is almost negligible. This implies that the HARE-deposited films contain fewer defects than their implanted counterparts. For HARE samples with an excess Si content of $20 \%$, the emission peak is weak and centered at $\sim 800 \mathrm{~nm}$. Emission at this wavelength is normally attributed to Si nanocrystals, implying that nanocrystals are present in as-deposited HARE films for high excess Si concentrations.

Following annealing at $1100{ }^{\circ} \mathrm{C}$, HARE and ionimplanted samples show a single broad PL emission peak centered at around 800-840 nm, consistent with the presence of Si nanocrystals [Fig. 2(b)]. The peak emission wavelength is known to depend on the size distribution of the Si nanocrystals and shifts to longer wavelengths (redshift) as the crystallites increase in size. ${ }^{4}$ The PL emission intensity is also expected to decrease for larger crystallites due to a reduction in the transition probability for exciton recombination as the crystallites become more bulk like. ${ }^{17}$ Figure 2(b) shows that the emission intensity is similar for the HARE sample containing 8 at. $\%$ excess $\mathrm{Si}$ and the ion-implanted sample containing 10 at. $\%$ excess Si. The spectrum from the HARE 


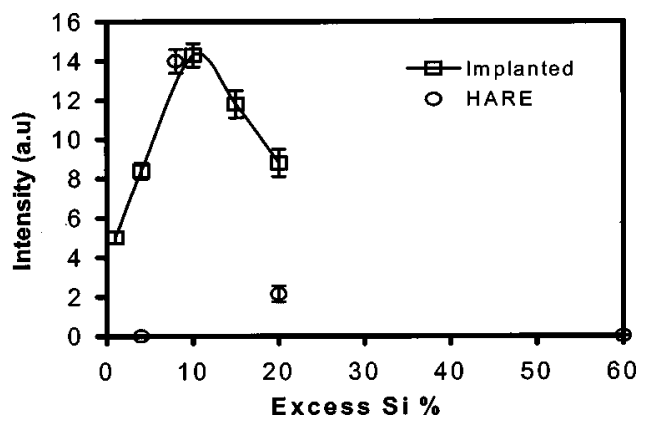

FIG. 3. PL emission intensity of the $800 \mathrm{~nm}$ peak as a function of excess $\mathrm{Si}$ concentration for HARE samples and implanted samples, annealed at $1100{ }^{\circ} \mathrm{C}$ in $\mathrm{N}_{2}$.

sample is slightly redshifted relative to the implanted sample, suggesting slightly larger nanocrystals in this case. The HARE sample has an 8 at. \% $\mathrm{Si}$ excess throughout its 500 $\mathrm{nm}$ thickness, corresponding to a total excess areal density of $2.6 \times 10^{17} \mathrm{Si} . \mathrm{cm}^{-2}$, slightly higher than that of the implanted sample which was implanted with $2.0 \times 10^{17} \mathrm{Si} / \mathrm{cm}^{-2}$. Implanted samples have a skewed Gaussian distribution of excess $\mathrm{Si}$ with a peak excess concentration of 10 at. \% and a full width at half maximum of approximately $400 \mathrm{~nm}$. A considerable fraction of the implanted volume therefore has an excess Si concentration less than 8 at. \%. The higher average concentration in the HARE sample would favor the formation of larger nanocrystals, consistent with the observed redshift.

Figure 3 compares the PL emission intensity for HARE and ion-implanted samples as a function of excess Si concentration. Clearly the trends are similar for both sample sets-the intensity initially increases with increasing Si concentration, reaches a maximum for an excess Si concentration of $\sim 8-10$ at. \%, and then decreases at higher concentrations. The intensity is expected to increase initially due to the increasing number of nanocrystals in the film. The reason for reduction in intensity at higher concentrations is less clear, however, there are several possibilities. For example. (a) the decreasing transition probability for larger crystallites, (b) the increasing probability for nanocrystal-nanocrystal interactions and the possibility of energy transfer to nonradiative crystallites, and (c) the increasing probability for defects in larger crystallites.

The oscillator strength can be shown ${ }^{17}$ to scale approximately at $d^{-2.25}$, where $d$ is the nanocrystal diameter. Hence a doubling in particle size is expected to result in a $\sim 4-5$ fold reduction in emission intensity. ${ }^{17}$ Dipole-dipole interactions provide a very efficient mean of transferring energy between closely spaced nanocrystals ${ }^{18}$ and since a large fraction of the large nanocrystals are optically inactive, ${ }^{18}$ such energy transfer would be expected to reduce the total emission intensity. For an excess Si concentration of 10 at. \%, and assuming that all of the excess atoms precipitate into nanocrystals of average size $3 \mathrm{~nm},{ }^{19}$ the interparticle separation is $\sim 2 \mathrm{~nm}$. Since the dipole-dipole interaction scales with $r^{-6,20}$ where $r$ is the interparticle separation, a rapid decrease in emission intensity is predicted with increasing particle concentration. Finally, the probability of a crystallite containing a defect is expected to scale with the crystal volume, producing a reduction in intensity that scales with $r^{-3}$. (NB: the influence of interface defects between the crystallite and surrounding oxide would scale with $r^{-2}$ ). Comparison with these models requires detailed particle sizing information and is complicated by the broad size distribution of nanocrystallites present in the samples. Further work is required before a meaningful quantitative comparison can be undertaken. However, it is clear that a reduction in emission intensity for high excess Si concentrations is expected.

In summary, it has been shown that HARE can be used to produce $\mathrm{Si}$-rich $\mathrm{SiO}_{2}$ layers suitable for the fabrication of Si-nanocrystal-rich films. As-deposited films were shown to contain a much lower concentration of optically active defects than similarly doped ion-implanted films. After suitable annealing, the HARE films exhibited strong nanocrystalbased luminescence similar to that from ion-implanted samples. The dependence of the luminescence intensity on excess Si concentration was also shown to be similar to that for ion-implanted films, initially increasing with increasing concentration before reaching a maximum and decreasing with concentration thereafter.

${ }^{1}$ L. T. Canham, Appl. Phys. Lett. 57, 1046 (1990).

${ }^{2}$ S. Guha, M. D. Pace, D. N. Dunn, and I. L. Singer, Appl. Phys. Lett. 70, 1207 (1997).

${ }^{3}$ S. Guha, J. Appl. Phys. 84, 5210 (1998).

${ }^{4}$ T. Inokuma, Y. Wakayama, T. Muramoto, R. Aoki, Y. Kurata, and S. Hasegawa, J. Appl. Phys. 83, 2228 (1998).

${ }^{5}$ H. Z. Song and X. M. Bao, Phys. Rev. B 55, 6988 (1997)

${ }^{6}$ T. S. Iwayama, K. Fujita, M. Akai, S. Nakao, and K. Saitoh, J. Non-Cryst. Solids 187, 112 (1995).

${ }^{7}$ D. Zhang, R. M. Kolbas, P. D. Milewski, D. J. Lichtenwalner, A. I. Kingon, and J. M. Zavada, Appl. Phys. Lett. 65, 2684 (1994).

${ }^{8}$ S. Tong, X. N. Liu, and X. M. Bao, Appl. Phys. Lett. 66, 469 (1995).

${ }^{9}$ T. S. Iwayama, S. Nakao, and K. Saitoh, Appl. Phys. Lett. 65, 1814 (1994).

${ }^{10}$ X. Zhao, O. Schoenfeld, J. Kusano, Y. Aoyagi, and T. Sugano, Jpn. J. Appl. Phys., Part 2 33, L649 (1994).

${ }^{11}$ Q. Zhang, S. C. Bayliss, and R. A. Hutt, Appl. Phys. Lett. 66, 1977 (1995).

${ }^{12}$ A. G. Nassiopoulos, S. Grigoropoulos, E. Gogolides, and D. Papadimitriou, Appl. Phys. Lett. 66, 1114 (1995).

${ }^{13}$ A. Durandet, R. Boswell, and D. McKenzie, Rev. Sci. Instrum. 66, 2908 (1995).

${ }^{14}$ S. P. Withrow, C. W. White, A. Meldrum, J. D. Budai, D. M. Hembree Jr., and J. C. Barbour, J. Appl. Phys. 86, 1 (1999).

${ }^{15}$ P. G. Pai, S. S. Chao, T. Tagaki, and G. Lucovsky, J. Vac. Sci. Technol. A 4, 689 (1986).

${ }^{16}$ T. Shimizu-Iwayama, K. Fujita, S. Nakao, K. Saitoh, T. Fujita, and N. Itoh, J. Appl. Phys. 75, 7779 (1996).

${ }^{17}$ F. Ranjan, V. A. Singh, and G. C. John, Phys. Rev. B 58, 1158 (1998).

${ }^{18}$ M. L. Brongersma, Ph.D. thesis, Utrecht University, The Netherlands, 1998.

${ }^{19}$ By using the volume fraction of nanocrystals: $F=N^{*} V$, the nanocrystals volume: $V=4 / 3^{*}\left(\pi R^{3}\right)$, we obtain the spacing between two nanocrystals as: $r=\left[(4 \pi / 3 F)^{1 / 3}-2\right]^{*} R$.

${ }^{20}$ Th. Förster, Comparative Effects of Radiation, edited by M. Burton, J. S. Kirby-Smith, and J. L. Magee (Wiley, New York, 1960), p. 301. 